\title{
Impact of smoking and smoking cessation on cardiovascular events and mortality among older adults: meta-analysis of individual participant data from prospective cohort studies of the CHANCES consortium
}

\author{
Ute Mons, ${ }^{1}$ Aysel Müezzinler, ${ }^{1,2}$ Carolin Gellert, ${ }^{1}$ Ben Schöttker, ${ }^{1}$ Christian C Abnet, ${ }^{3}$ Martin Bobak, ${ }^{4}$ \\ Lisette de Groot, ${ }^{5}$ Neal D Freedman, ${ }^{3}$ Eugène Jansen, ${ }^{6}$ Frank Kee, ${ }^{7}$ Daan Kromhout, ${ }^{5}$ \\ Kari Kuulasmaa, ${ }^{8}$ Tiina Laatikainen, 8, 9, 10 Mark G O’Doherty, ${ }^{7}$ Bas Bueno-de-Mesquita, 11, 12, 13, 14 \\ Philippos Orfanos, ${ }^{15,}{ }^{16}$ Annette Peters, ${ }^{17,} 18$ Yvonne T van der Schouw, ${ }^{19}$ Tom Wilsgaard, ${ }^{20}$ \\ Alicja Wolk, ${ }^{21}$ Antonia Trichopoulou, ${ }^{15,16}$ Paolo Boffetta, ${ }^{15,}, 22$ Hermann Brenner, ${ }^{1}$ on behalf of the \\ CHANCES consortium
}

\begin{abstract}
For numbered affiliations see end of article.

Correspondence to: $U$ Mons, Division of Clinical

Epidemiology and Aging Research, German Cancer Research Center (DKFZ), Im Neuenheimer Feld 581, 69120 Heidelberg, Germany

u.mons@dkfz.de

Cite this as: $B M$ J 2015;350:h1551 doi:10.1136/bmj.h1551
\end{abstract}

Accepted: 6 February 2015

\section{ABSTRACT}

OBJECTIVE

To investigate the impact of smoking and smoking cessation on cardiovascular mortality, acute coronary events, and stroke events in people aged 60 and older, and to calculate and report risk advancement periods for cardiovascular mortality in addition to traditional epidemiological relative risk measures.

\section{DESIGN}

Individual participant meta-analysis using data from 25 cohorts participating in the CHANCES consortium. Data were harmonised, analysed separately employing Cox proportional hazard regression models, and combined by meta-analysis.

\section{RESULTS}

Overall, 503905 participants aged 60 and older were included in this study, of whom 37952 died from cardiovascular disease. Random effects meta-analysis of the association of smoking status with cardiovascular mortality yielded a summary hazard ratio of 2.07 ( $95 \% \mathrm{Cl} 1.82$ to 2.36$)$ for current smokers and 1.37 (1.25 to 1.49) for former smokers compared with never smokers. Corresponding summary estimates for risk advancement periods were 5.50 years ( 4.25 to 6.75$)$ for current smokers and 2.16 years (1.38 to 2.39) for former smokers. The excess risk in smokers increased with cigarette consumption in a

\section{WHAT IS ALREADY KNOWN ON THIS TOPIC}

Generally, smoking is a major modifiable risk factor for disease and death, and smoking cessation is beneficial in reducing smoking related risks

However, even though most cardiovascular events occur in older adults, this age group has been understudied when it comes to the cardiovascular risks of smoking and the potential benefits of smoking cessation on cardiovascular health

\section{WHAT THIS STUDY ADDS}

Using data from a large consortium of cohorts from all over Europe and the United States, we corroborated and expanded evidence from previous studies to show that smoking is a strong independent risk factor for cardiovascular events and mortality among older adults. We demonstrated that even at older ages, smoking cessation is beneficial in reducing the cardiovascular excess risk caused by smoking Hence, given the increasing numbers of older people and the higher incidence of cardiovascular events and mortality at older age, there is tremendous potential for smoking and cardiovascular disease prevention in this group of people dose-response manner, and decreased continuously with time since smoking cessation in former smokers. Relative risk estimates for acute coronary events and for stroke events were somewhat lower than for cardiovascular mortality, but patterns were similar.

\section{CONCLUSIONS}

Our study corroborates and expands evidence from previous studies in showing that smoking is a strong independent risk factor of cardiovascular events and mortality even at older age, advancing cardiovascular mortality by more than five years, and demonstrating that smoking cessation in these age groups is still beneficial in reducing the excess risk.

\section{Background}

In 1964, the first of a series of the US Surgeon General's reports on the health consequences of smoking concluded that male smokers had a higher death rate from coronary heart disease than non-smoking males, but evidence was yet not sufficient to conclude a causal relation. ${ }^{1}$ In the 50 years since the publication of this landmark report, ever stronger epidemiological evidence for a causal link between tobacco smoking and cardiovascular diseases and mortality has accrued from a multitude of prospective cohort studies. ${ }^{23}$ During the same decades cardiovascular mortality rates have been decreasing in developed countries, as have rates of cigarette consumption, but cardiovascular disease remains a leading cause of death. ${ }^{4}$

The incidence of cardiovascular disease increases with age and most events occur in older adults. ${ }^{5}$ Given current demographic trends, prevention in older adults through risk factor management is of crucial importance to reduce the burden of cardiovascular disease. But despite being one of the major modifiable risk factors, few prospective studies have specifically investigated the effect of smoking ${ }^{6-12}$ and smoking cessation ${ }^{78}$ on cardiovascular outcomes at advanced age. These studies suggest that even in later life, smoking is a risk factor for cardiovascular deaths and disease, and that smoking cessation could still be beneficial.

Communicating the risks of smoking and the benefits of quitting to smokers could be an effective means to promote cessation. While relative risks might be especially difficult to grasp for lay people, risk advancement 
periods have been proposed to be of particular use in risk communication. ${ }^{1314}$ The risk advancement period (RAP) gives the average time by which the occurrence of an event (such as disease incidence or death) due to a risk factor is advanced in exposed people compared with unexposed people. ${ }^{15}$

With this work, we sought to provide evidence of the impact of smoking and smoking cessation on cardiovascular outcomes in people aged 60 and older, in a meta-analysis of individual participant data from cohort studies from Europe and the United States participating in the CHANCES consortium (Consortium on Health and Ageing: Network of Cohorts in Europe and the United States). In addition to traditional epidemiological relative risk measures, we also calculated and report the risk advancement period for cardiovascular mortality.

\section{Methods}

\section{Study design and participants}

This study was conducted within the CHANCES consortium (http://www.chancesfp7.eu/). ${ }^{16}$ This collaborative project aims at combining and integrating data from cohort studies conducted in Europe and North America in order to study major age related chronic conditions and to produce evidence on health determinants in older adults. The consortium includes 15 population based cohort studies, including several multicentre studies, with participants from 24 countries. The data from these cohort studies were combined by harmonising variables of interest according to pre-agreed and consented harmonisation rules.

For this study, we used data from 10 cohort studies and cohort consortia of the CHANCES consortium and additionally from two cohort studies with open data access (ELSA, NHANES III), adding up to 25 different cohorts with participants from 23 countries (see table 1 for an overview, with detailed descriptions of these cohort studies provided in the appendix). Because there have been only a few studies on the cardiovascular risks of smoking in older age, we focused our study on older adults and included only people aged 60 and older.

All CHANCES cohort studies are conducted in accordance with the Declaration of Helsinki. For each study, investigators satisfied the local requirements for ethical research, including obtaining informed consent from participants.

\section{Outcomes}

Three cardiovascular outcomes were examined in this study. As primary outcome, cardiovascular deaths were defined as fatal cardiovascular events according to cause of death (that is, ICD-8, codes 390-458; ICD-9, codes 390-459; ICD-10, codes I00-I99). Cause of death was obtained from death registers or from death certificates. The only exception was the SHARE cohort, for which cause of death was ascertained through an "end of life interview" with a household member or another close contact of the deceased person. Secondary outcomes were incidence of acute coronary events and stroke. For the incidence analyses people were excluded who had a history of acute coronary events or stroke before inclusion in the study. Acute coronary events comprised confirmed fatal and non-fatal coronary events including acute myocardial infarction, unstable angina pectoris or coronary death according to pertinent definitions used in the respective cohort studies (such as WHO definition ${ }^{17}$ or WHO MONICA criteria ${ }^{18}$ ). Stroke events comprised fatal or non-fatal acute events that fulfilled the typical symptoms of stroke (that is, people presenting clinical signs and symptoms suggestive of subarachnoid haemorrhage, intracerebral haemorrhage, or cerebral infarction). Both acute coronary and stroke events were identified through linkage to registries, contact with a physician, through follow-up questionnaires (self report), or interviews with close relatives. If a case was identified through self report or interview with a relative, validation was achieved through contact with the participant's physician, or by retrieving medical records, hospital discharge diagnoses, death certificates, or autopsy reports.

All included cohort studies provided data on cardiovascular deaths; however, non-fatal acute coronary and stroke events were not available for MORGAM SHIP Greifswald, NHANES, NIH-AARP, SENECA, or SHARE.

\section{Exposure variables}

All included cohort studies collected data on current smoking status at baseline using interviews or self administered questionnaires, and most studies also collected data on smoking intensity and smoking cessation (see supplementary table 1 for an overview of availability of variables). Current smoking status describes the status of never, former, or current smoking of tobacco products at the time of the baseline interview. Current smoking was regular or daily smoking, according to the cohort study's definition. Never smoking was either defined as never having smoked daily or regularly or as not having smoked more than 100 cigarettes over a lifetime. Current intensity of smoking was assessed as the average consumption of cigarettes per day. Smokers with available information on cigarette smoking intensity were categorised into the following categories: smoking < 10 cigarettes per day, 10-19 cigarettes per day, $\geq 20$ cigarettes per day. Former smokers were asked for age or date of smoking cessation and, according to the time since smoking cessation at baseline, were categorised into the following categories: stopped smoking < 5 years ago, 5-9 years ago, 10-19 years ago, $\geq 20$ years ago.

\section{Covariates}

Covariates comprised sex, age, education, alcohol consumption, body mass index (BMI), physical activity, history of diabetes, total cholesterol, and systolic blood pressure. All of these variables were measured at baseline. Education was categorised into low, moderate, and high, which was either based on the highest level of education (low: primary education or less; moderate: more than primary but less than college or university education; high: college or university education), or on the number of years in full time education which was 


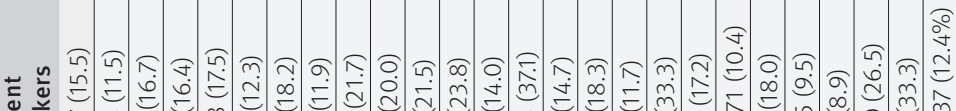

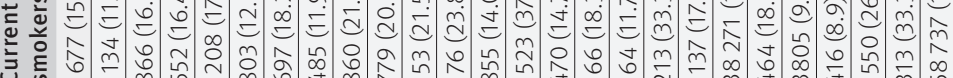
等

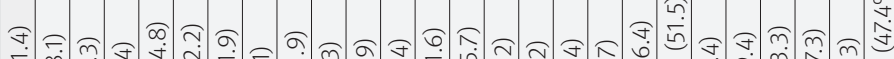
竞

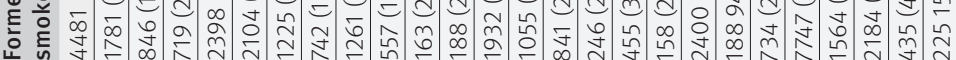

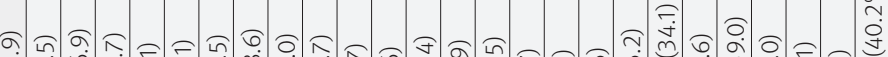

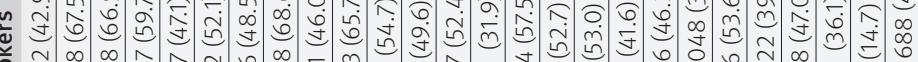

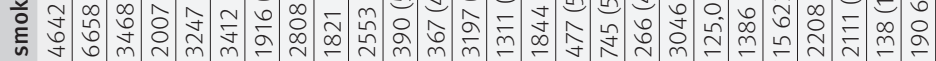

๙mळ ๑

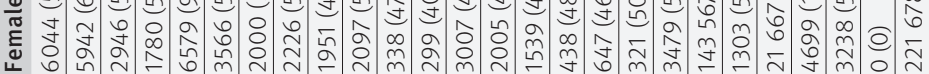

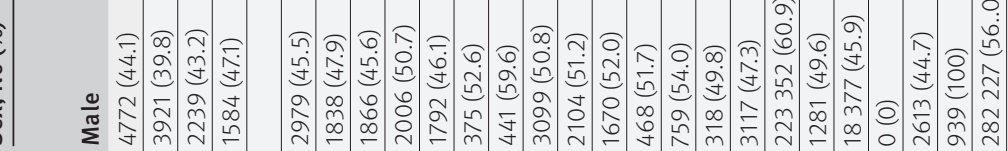

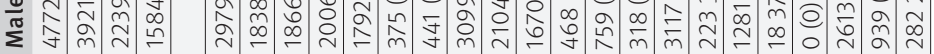

๙

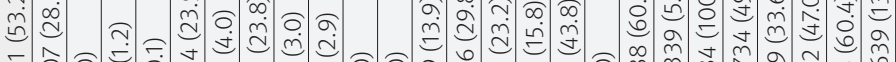

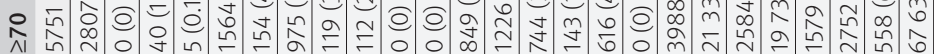

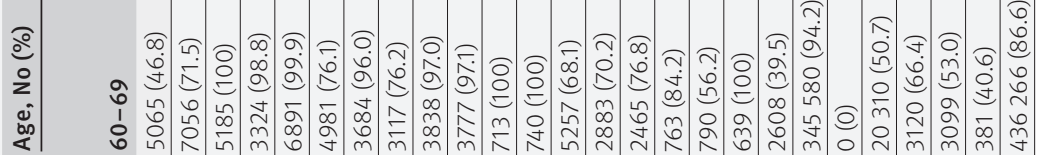

n+

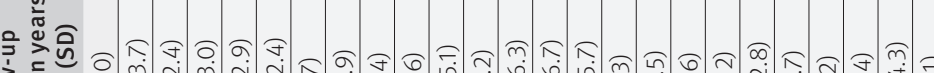

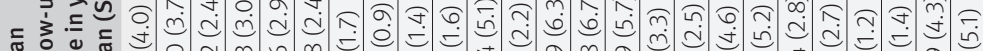

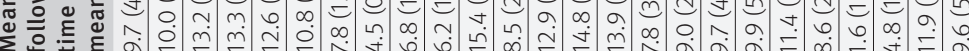

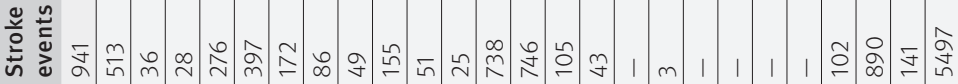

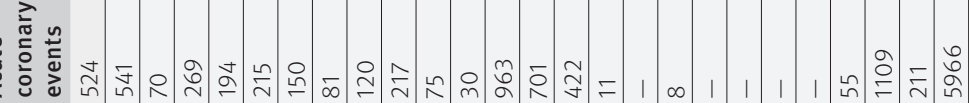
’े

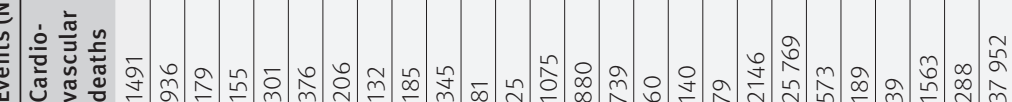

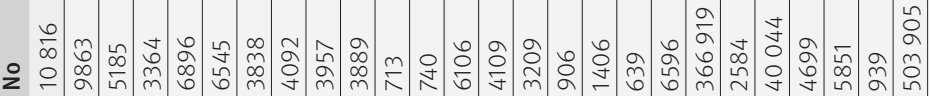

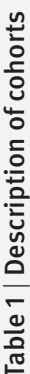

then translated into categories (low: $<10$ years of education; moderate: 10 or 11 years; high: $\geq 12$ years). Alcohol consumption was assessed as average daily alcohol intake per day (g) and categorised into three categories based on sex specific cut-offs: non-drinker ( $0 \mathrm{~g}$ of alcohol per day), moderate drinker (women: $\geq 0 \mathrm{~g}$ to $<10 \mathrm{~g}$ per day; men: $\geq 0 \mathrm{~g}$ to $<20 \mathrm{~g}$ per day), and heavy drinker (women: $\geq 10 \mathrm{~g}$ per day, men: $\geq 20 \mathrm{~g}$ per day). ${ }^{19}$ For SHARE three categories were defined on the basis of the usual drinking pattern according to the number of days per week on which alcohol was drunk (non-drinker: self reported non-drinking; moderate drinker: drinking on $\leq 2$ days per week; heavy drinker: drinking on $\geq 3$ days per week). BMI was computed as body weight in kilograms divided by squared body height in metres, with weight and height having been assessed either through self report or through measurement. BMI was categorised into normal weight (BMI of $\leq 25 \mathrm{~kg} / \mathrm{m}^{2}$ ), overweight $\left(25\right.$ to $\left.<30 \mathrm{~kg} / \mathrm{m}^{2}\right)$, and obese $\left(\geq 30 \mathrm{~kg} / \mathrm{m}^{2}\right)$. Physical activity was coded as a binary variable indicating whether participants engage in vigorous physical activity at least once per week. History of diabetes was a binary variable that was based either on self report of participants, on validated information from their physicians, diabetes registers or medical records, or on results of blood measurements (such as 2 hour plasma glucose $\geq 200 \mathrm{mg} / \mathrm{ml}$ in an oral glucose tolerance test, $\mathrm{HbA}_{1 \mathrm{c}} \geq 6.5 \%$, or fasting plasma glucose $\geq 126 \mathrm{mg} / \mathrm{dl}$ ). Total cholesterol ( $\mathrm{mmol} / \mathrm{l})$ was measured from serum or plasma; if plasma was used, values were converted into serum values. Systolic blood pressure $(\mathrm{mm} \mathrm{Hg}$ ) was either measured in a baseline examination, or obtained from other sources of information such as medical records or through participants' physicians. Both total cholesterol and systolic blood pressure were used as continuous variables.

\section{Statistical analyses}

For the multivariable analyses, multiple Cox proportional hazards regression models were employed to estimate hazard ratios and $95 \%$ confidence intervals for the associations of the smoking related predictors with the three different cardiovascular outcomes. This was done separately for each cohort by using uniform analysis scripts. If a cohort consisted of sub-cohorts from different survey periods (as in the case of all MORGAM cohorts except SHIP Greifswald) or was a multicentre study (SHARE, SENECA), then a stratified Cox model was employed allowing the baseline hazard to vary between strata-that is, across sub-cohorts or centres, respectively. The proportional hazards assumption was assessed graphically for each cohort. Three models with different sets of adjustments were fitted. The simple model was adjusted for sex and age. The main model was additionally adjusted for education, alcohol consumption, BMI, and physical activity. However, for a few cohorts, not all variables were available or could not be harmonised according to the CHANCES variable harmonisation rules. In particular, harmonised variables for alcohol consumption were not available for MORGAM Northern Sweden and for NHANES 
(in NHANES information on alcohol consumption was only collected in the subpopulation of those participating in the medical examination), and harmonised variables for physical activity were not available for the MORGAM cohorts and for EPIC-Elderly Sweden (see supplementary table 1 for an overview). In those cases, the main model was fitted without this particular variable. An additional model was further adjusted for history of diabetes, total cholesterol, and systolic blood pressure in addition to the covariates included in the main model. The rationale for this analysis was to explore if and to what extent the observed associations might be mediated by diseases for which smoking is a well known risk factor. ${ }^{2}$ However, these variables were not available for all cohorts.

We used the results of the multivariable Cox models to calculate risk advancement periods and their 95\% confidence intervals, which are reported for the primary outcome in addition to hazard ratios. Risk advancement periods are calculated as the ratio of the regression coefficients of the respective predictor variable and age (see appendix for further details and formulas) and quantify by how many years cardiovascular mortality is advanced in exposed participants compared with the unexposed reference group (for example, current smokers in comparison with never smokers). ${ }^{15}$ In a few cases, if the cohorts had only a very narrow age range that did not offer enough variation to compute reliable estimates of the regression coefficients for age, the resulting risk advancement periods would not be meaningful and were therefore not calculated.

After the estimation of hazard ratios and risk advancement periods for the single cohorts, meta-analyses were applied in order to calculate summary hazard ratios and risk advancement periods estimates across cohorts. We computed both fixed effects models, and random effects models using the DerSimonian-Laird method. ${ }^{20}$ Owing to substantial heterogeneity across cohort results as assessed with $\mathrm{I}^{2}$ and $\mathrm{Q}$ statistics, ${ }^{21}$ random effects estimates are reported as the main results, since random effects models account for variability of effects across individual studies. We explored age and sex differences by subgroup analyses. Several sensitivity analyses (especially through exclusion of studies) were conducted to assess the robustness of the summary estimates. Dose-response relations were assessed by meta-analysis for dose-response data using the Greenland and Longnecker method ${ }^{22}$ and a random effects model as implemented in the SAS\%metadose macro. $^{23}$

All statistical tests were two sided, with an alpha level of 0.05. SAS v.9.3 was used for the descriptive statistics, the survival analyses, and the dose-response meta-analysis. R v.3.0.2 and the package 'meta' ${ }^{24}$ were used to carry out the main individual participant meta-analyses.

\section{Results}

In total, 503905 participants aged 60 and older were included in this study, with 37952 cardiovascular deaths recorded during follow-up (table 1). NIH-AARP was the largest study, contributing 366919 participants and 25769 cardiovascular deaths. The mean follow-up time was between approximately 8 and 13 years for most of the studies, with the noteworthy exception of SHARE with a substantially shorter mean follow-up of just 1.6 years. About half of the cohorts had substantial proportions of participants equal to or above the age of 70 at baseline (for example, ELSA, NHANES, SHARE, Tromsø, Zutphen), while the other half consisted exclusively or predominantly of adults below that age, including the biggest study NIH-AARP. Altogether, only $13.4 \%$ of the sample (67639) was 70 years or older, but 22.8 of all deaths (8638) occurred in this age group . Two cohorts comprised only participants of one sex, SMC (only women) and Zutphen (only men). Overall, $44 \%$ of the meta-analysed sample were female (221678). Ninety four per cent (474583) of all participants had reported their current smoking status. Among these, smoking prevalence varied widely, from $8.9 \%$ in the SMC cohort to $37.1 \%$ in MORGAM Glostrup. Overall, 40.2\% (190688) of participants were self reported never smokers, 47.4\% (225158) were former smokers, and 12.4\% (58737) were current smokers.

Meta-analysis of the association of smoking status with cardiovascular mortality yielded a summary hazard of 2.07 (95\% confidence interval 1.82 to 2.36 ) for current smokers and of 1.37 (1.25 to 1.49) for former smokers compared with never smokers (fig 1). Corresponding summary estimates for risk advancement periods were 5.50 (4.25 to 6.75) for current and 2.16 (1.38 to 2.39) for former smokers (fig 2). The excess risk in smokers increased with cigarette consumption; the highest hazard ratio of 2.63 (2.28 to 3.04) and the corresponding risk advancement period estimate of 6.90 (5.59 to 8.20) were found in current smokers who smoked 20 or more cigarettes per day (fig 3; supplementary figs 3 and 4). The trend of risk increase per 10 cigarettes was 1.40 (1.33 to 1.47, $\mathrm{P}<0.001$; details not shown). In former smokers, the smoking related cardiovascular mortality risk decreased continuously with time since smoking cessation with a hazard ratio for trend per 10 years of 0.85 (0.82 to $0.89, \mathrm{P}<0.001$; details not shown). Participants who had stopped smoking less than 5 years before baseline had a reduced hazard ratio for cardiovascular mortality of 0.90 (0.81 to 1.00), which translated to a risk advancement period estimate of -0.82 ( -1.72 to 0.07$)$ compared with continuing smokers. Corresponding hazard ratios and risk advancement period estimates for participants having quit 5-9 years ago were 0.84 (0.73 to 0.95 ) and -1.34 ( -2.29 to -0.39$)$, were 0.78 (0.71 to $0.85)$ and -1.96 ( -2.69 to -1.24$)$ for those having quit 10 to 19 years ago, and were 0.61 (0.54 to 0.69$)$ and -3.94 ( -4.86 to -3.03$)$ for those having quit 20 or more years ago (fig 3; supplementary figs 5 and 6). A similar pattern of decreasing excess risk was seen when the cardiovascular mortality risk was modelled in reference to never smokers with a hazard ratio for trend of 0.82 per 10 years ( 0.78 to $0.86, \mathrm{P}<0.001$; details not shown). Former smokers who had stopped smoking less than 5 years ago had a hazard ratio of 1.74 (1.51 to 2.01), which decreased in a dose-response manner with categories of 


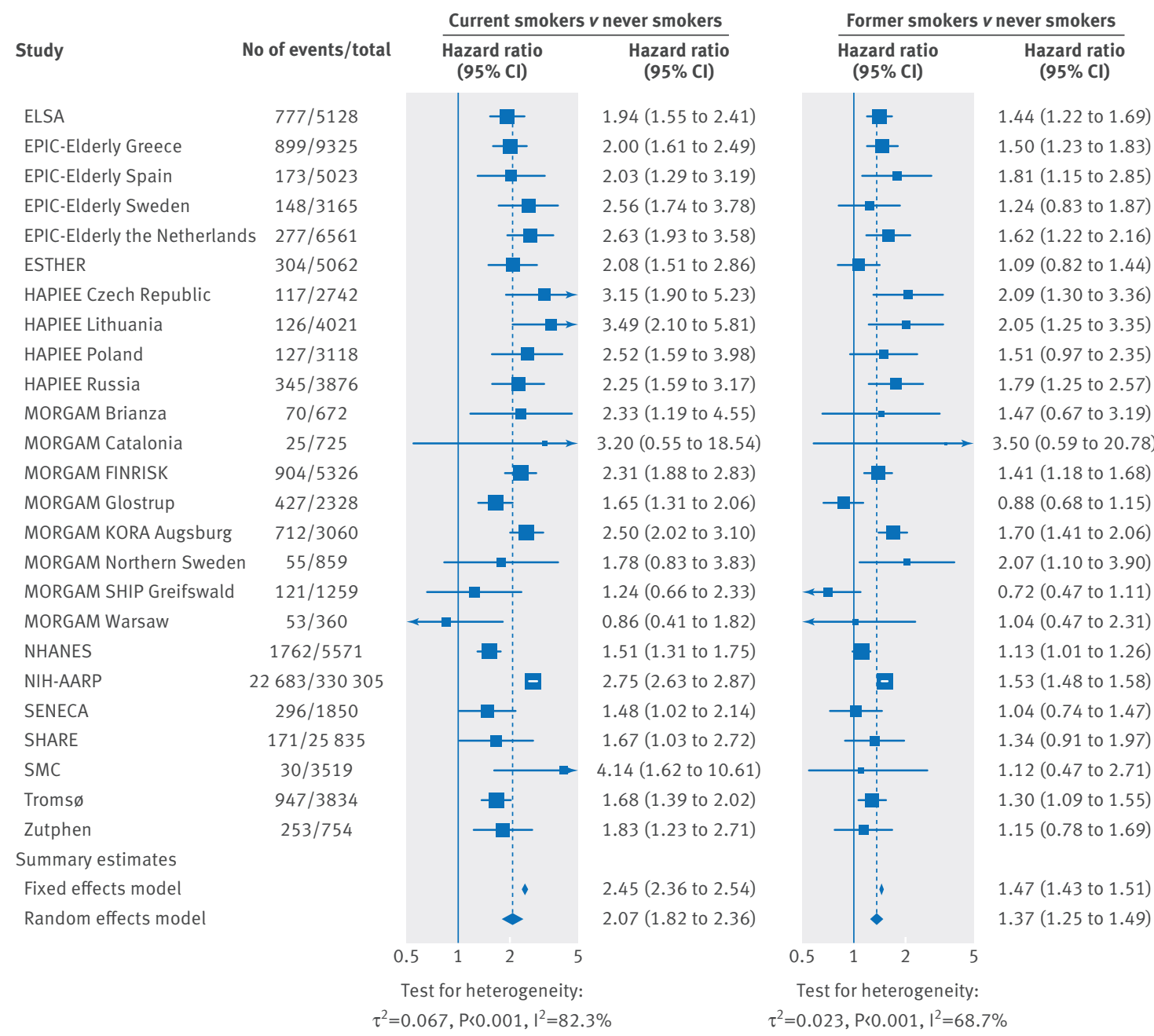

Fig 1 | Meta-analysis of the association of current smoking status with cardiovascular mortality

time since smoking, and those who had stopped smoking 20 or more years ago only had a slightly increased hazard ratio of 1.15 (1.02 to 1.30). The risk advancement period estimates decreased accordingly with categories of time since smoking cessation from 3.75 (2.78 to 4.71) in former smokers who had quit less than 5 years ago to a merely insignificantly elevated risk advancement period of 0.79 ( -0.12 to 1.69$)$ in those who had quit 20 or more years ago (fig 3; supplementary figs 9 and 10).

Further sex and age stratified analyses of the association of time since smoking cessation with cardiovascular mortality revealed quite consistent patterns across sex and age (fig 4; supplementary figs 7 and 8). Even though the dose-response relation of time since smoking cessation with cardiovascular mortality seems somewhat weaker in those aged 70 and older than in those aged 60 to 69, we still observed a clear decline of the risk with time since smoking cessation even in the oldest age group.

Similar patterns to those for cardiovascular mortality were seen for the associations of smoking related predictors with acute coronary and stroke events, albeit somewhat weaker, particularly in the case of stroke events (fig 5; supplementary figs 11, 14, 15, 16, 19, 20).
Hazard ratios for acute coronary events were 1.98 (1.75 to 2.25) for current smokers and 1.18 (1.06 to 1.32) for former smokers. The respective hazard ratios for stroke were 1.58 (1.40 to 1.78 ) and 1.17 (1.07 to 1.26). For both acute coronary and stroke events, risks tended to increase with daily cigarette consumption in current smokers (hazard ratio for linear trend per 10 cigarettes were: 1.36 (1.28 to $1.45, \mathrm{P}<0.001$ for acute coronary events, and 1.25 (1.19 to 1.31), $\mathrm{P}<0.001$ for stroke; details not shown), and to decrease with time since smoking cessation in former smokers (hazard ratios for linear trend per 10 years were: 0.83 ( 0.78 to 0.89 ), $\mathrm{P}<0.001$ for acute coronary events, and 0.87 (0.84 to 0.91 ), $\mathrm{P}<0.001$ for stroke; details not shown).

Summary estimates from age and sex stratified analyses for smoking status are reported in table 2 (see also supplementary figs 1, 2, 12,13,17, 18). Age and sex specific patterns were quite similar across outcomes. Risk estimates were somewhat higher in women compared with men; this difference was most pronounced for the risk of acute coronary events in current smokers, which was 2.26 (1.98 to 2.59) in women and 1.80 (1.51 to 2.15) in men. Hazard ratios also tended to be higher for 60 to 69 year old participants compared with those aged 70 or older. 


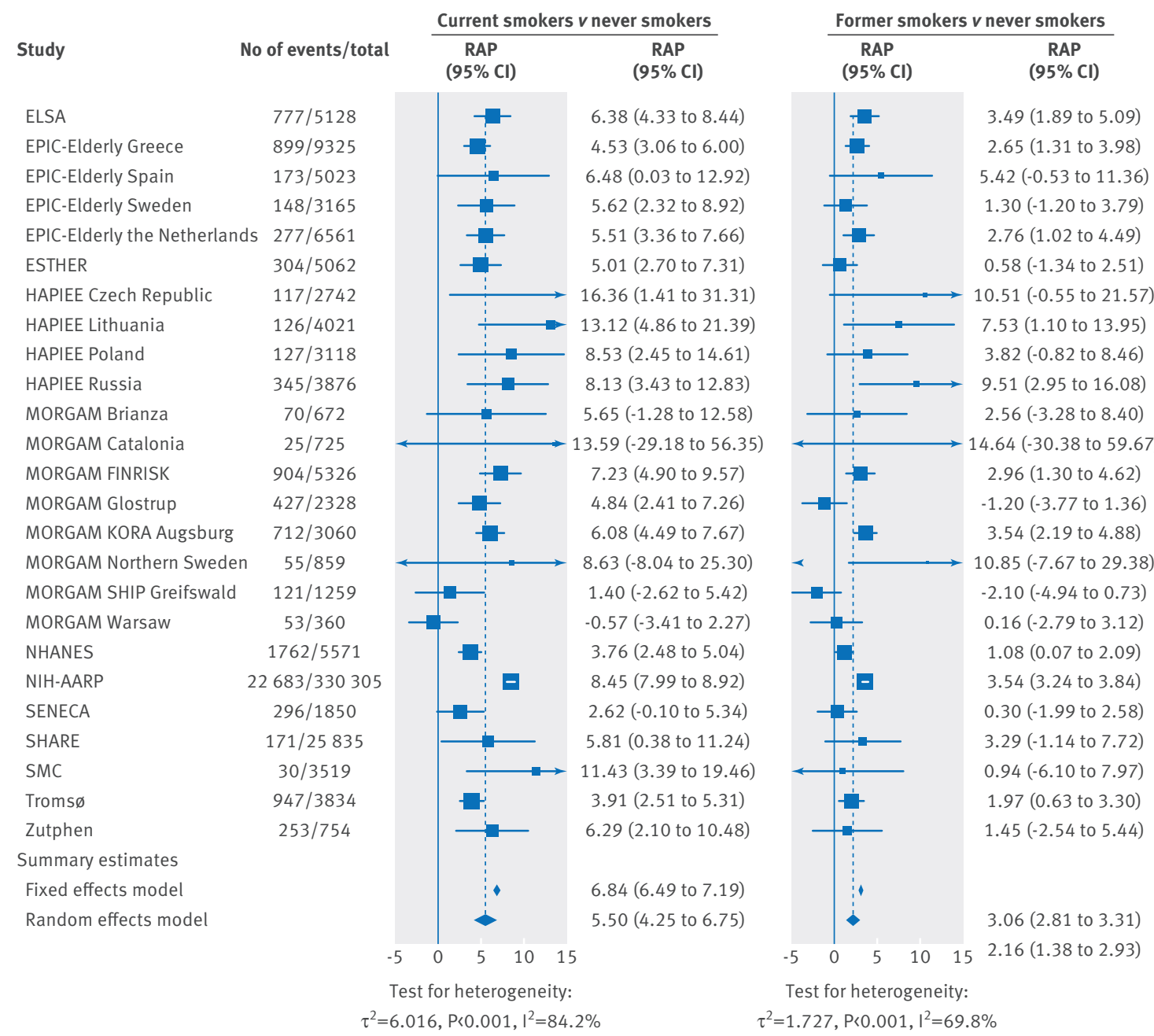

Fig 2 | Meta-analysis of risk advancement periods (RAP) for current smoking status and cardiovascular mortality

The greatest age difference was observed for the risk of cardiovascular mortality in current smokers, which was 2.45 (2.22 to 2.69) in 60 to 69 year olds compared with 1.70 (1.42 to 2.04 ) in those aged 70 or older.

Sensitivity analyses for meta-analyses excluding those studies for which physical activity or alcohol consumption information was not available as covariates showed very similar results (deta not shown). Further analyses included additional adjustment for history of diabetes, blood pressure, and total cholesterol. However, adding these covariates did not change the summary estimates to any relevant extent (details not shown).

\section{Discussion}

Principal findings

To our knowledge, the present work is currently the largest and most comprehensive study on the association of smoking with cardiovascular disease and mortality in older adults. We drew on harmonised and uniformly analysed data from a number of cohorts from Europe (covering eastern, northern, southern, western, and central Europe) and the US, and combined these studies by individual participant data meta-analyses.
Our results show that in people aged 60 years and older, smoking strongly contributes to acute coronary events, stroke, and cardiovascular deaths. Smokers had twofold hazards of cardiovascular mortality compared with never smokers, which, in terms of risk advancement periods, advanced the risk of dying from cardiovascular disease by 5.5 years. The hazard of incident acute coronary events for smokers was also twofold, and 1.5-fold for stroke events. Among smokers, the excess risk increased with higher levels of cigarette consumption. The increased excess risk among former smokers declined with time after smoking cessation in a dose-response manner.

\section{Comparison with other studies}

Only a few previous prospective studies on the association of smoking with cardiovascular outcomes included older adult populations, ${ }^{6-11}$ and even fewer focused specifically on people above the age of $60 .^{6-8}$ Our findings are generally in line with their results. For instance, an Australian study in men aged 69 and older found a hazard ratio of 1.70 (1.19 to 2.42) for a combined endpoint of stroke and myocardial infarction. ${ }^{6}$ A large Japanese study yielded relative cardiovascular mortality risks of 

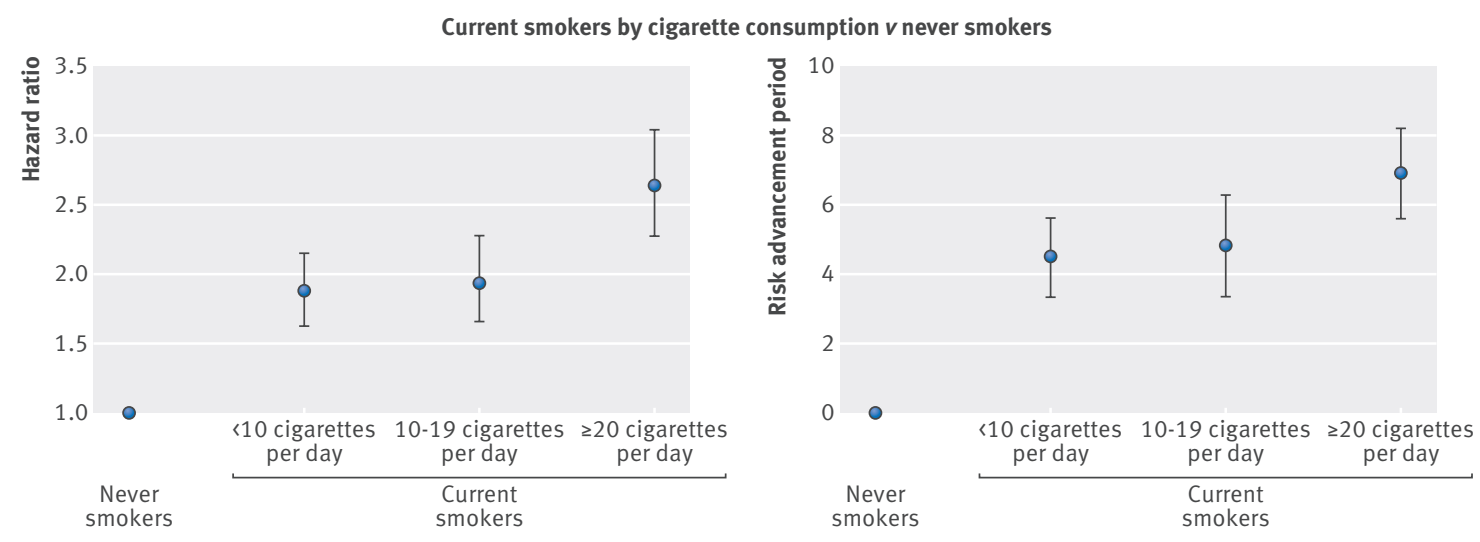

Former smokers by time since smoking cessation $v$ current smokers
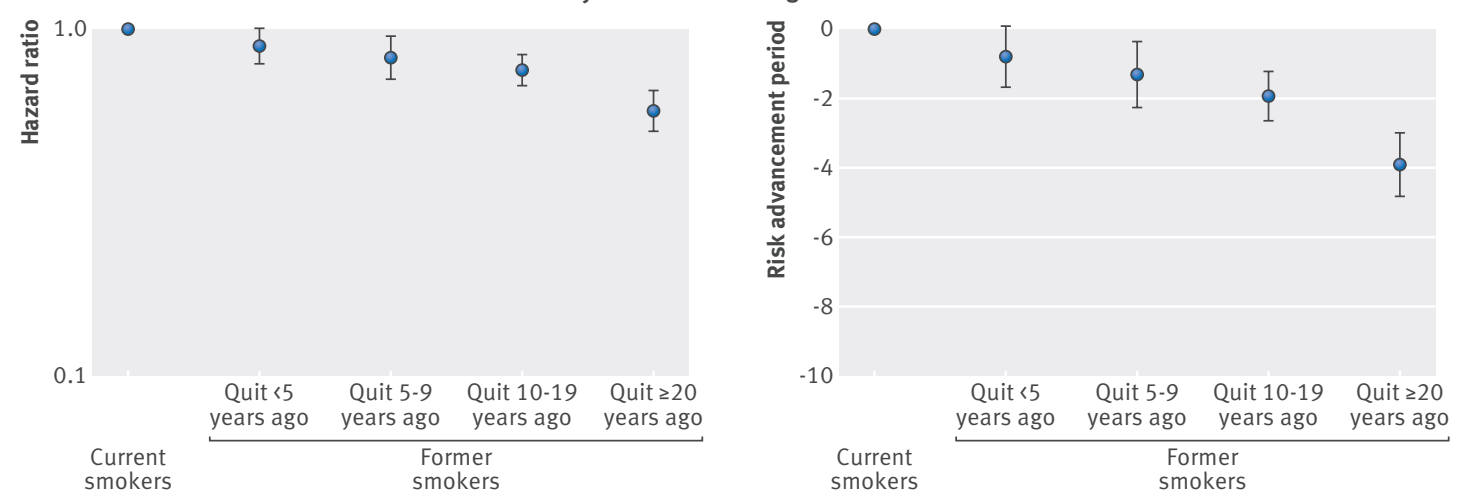

Former smokers by time since smoking cessation $\boldsymbol{v}$ never smokers
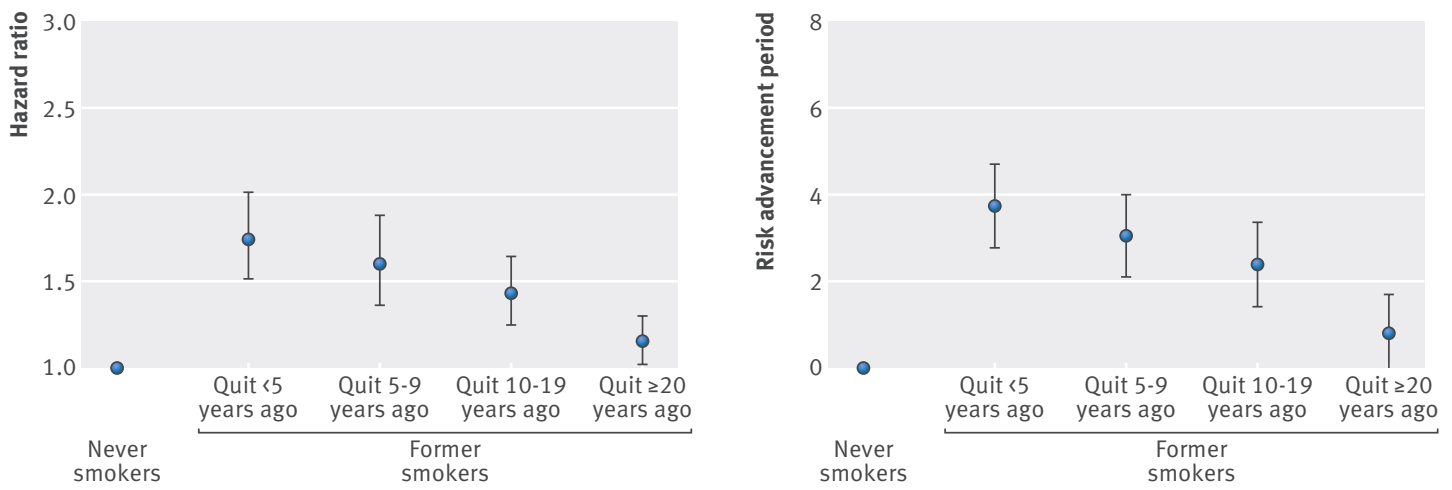

Fig 3 | Cardiovascular mortality summary estimates (random effects model) of hazard ratios and risk advancement periods for categories of cigarette consumption and time since smoking cessation

1.41 (1.19 to 1.67$)$ for male smokers and of 1.69 (1.32 to 2.15) for women aged 65 to 79 years over 10 years of follow-up. ${ }^{8}$ This study also observed a decline of the risk within a few years after smoking cessation, which is comparable with our findings. Only one previous study on the impact of smoking on cardiovascular outcomes reported risk advancement periods in addition to traditional risk measures. ${ }^{7}$ This study from Germany used data from the ESTHER cohort, which is also included in this present work (albeit with a longer follow-up time of 13 years and with older participants excluding those younger than 60). In the age group of 60 to 74 , a hazard ratio of 2.50 (1.74 to 3.61) for cardiovascular mortality and a corresponding risk advancement period estimate of 7.8 ( 4.3 to 11.3 ) years over 10 years of follow-up were reported, which is somewhat higher than the estimates observed in this present work but the dose-response patterns are consistent.

Our results on the benefits of smoking cessation are particularly noteworthy because so far the impact of cessation on cardiovascular mortality and disease risks have rarely been studied in older adults. Even though the risk avoided by smoking cessation is greater the earlier a smoker quits, ${ }^{3}$ our data show that smoking cessation was still associated with a substantial reduction of cardiovascular risks even in the oldest age groups. The hazard ratio of cardiovascular mortality was significantly reduced to 0.84 compared to continuing smokers already after five years since cessation. And within 20 years after smoking cessation, the excess risk compared to 


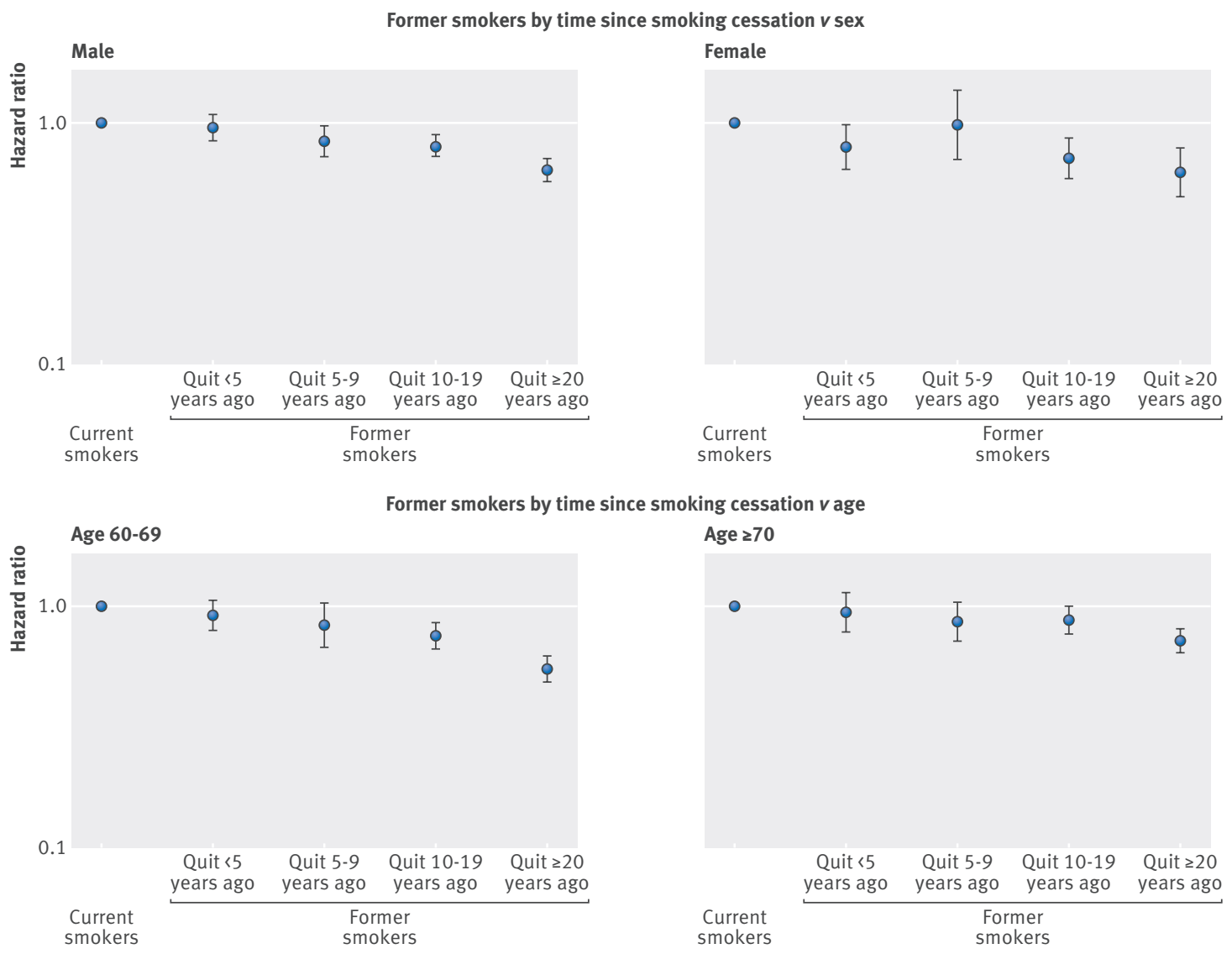

Fig 4 | Cardiovascular mortality summary estimates (random effects model) of hazard ratios for categories of time since smoking cessation by sex and age

never smokers reduced to an only slightly elevated hazard ratio of 1.15 . Thus, and in contrast to common misperceptions that older adult smokers might be too old to benefit from quitting, our findings indicate that smoking cessation should be encouraged at any age.

The excess risk in current and former smokers for adverse cardiovascular outcomes was still substantial in those aged 70 and older, but the relative association was weaker compared with those aged 60 to 69 . This is in line with findings from a British case-control study showing that the association of smoking and myocardial infarction weakens with every 10 year age group. ${ }^{11}$ One explanation for this phenomenon could be the "depletion of susceptibles" effect: ${ }^{25}$ owing to their increased mortality risks, a depletion of the number of higher risk smokers occurs while the healthier or lower risk people remain, leading to an attenuation of associations. Competing risks in smokers owing to increased hazards for other serious diseases caused by smoking might also play a role. ${ }^{26}$ But even though the impact of smoking appears to be somewhat weaker at older ages in relative terms, it may still be as large or even larger in absolute terms given the strong rise of incidence and mortality from cardiovascular diseases with age.

Richard Peto has coined the phrase "If women smoke like men, they die like men" when commenting on the similarly high smoking related all cause mortality risks in women compared with men. ${ }^{27}$ For adverse cardiovascular outcomes, our findings indicate that female smokers above the age of 60 have even somewhat higher relative risks than men. This is in accordance with findings from a recent meta-analysis of 75 prospective cohort studies that revealed that women had a $25 \%$ higher risk for coronary heart disease conferred by smoking compared with men. ${ }^{28}$ A similar meta-analysis on stroke pooled results from 25 Western cohorts and found that women had a 10\% higher risk for stroke compared with men. ${ }^{29}$ Whether such differences are result of biological differences or other mechanisms is unclear, ${ }^{28} 29$ but the female to male ratio of the smoking related hazard ratios in our study were of comparable magnitude for all cardiovascular outcomes.

\section{Limitations and strengths}

Even though the risk estimates appear remarkably consistent across the single cohorts, $\mathrm{I}^{2}$ and $\mathrm{Q}$ statistics of our meta-analyses suggest high and significant heterogeneity. The $\mathrm{I}^{2}$ describes the percentage of total variability across studies that is due to heterogeneity rather than chance, ${ }^{21}$ which for instance was $82.3 \%$ (and $68.7 \%$ ) in the meta-analyses on the association of current (and former) smoking with cardiovascular mortality. Apart from differences in study design, the variation in age distributions between cohorts could be one reason for the large heterogeneity. Indeed, age stratification reduced the $\mathrm{I}^{2}$ measure substantially to $40.8 \%$ for 

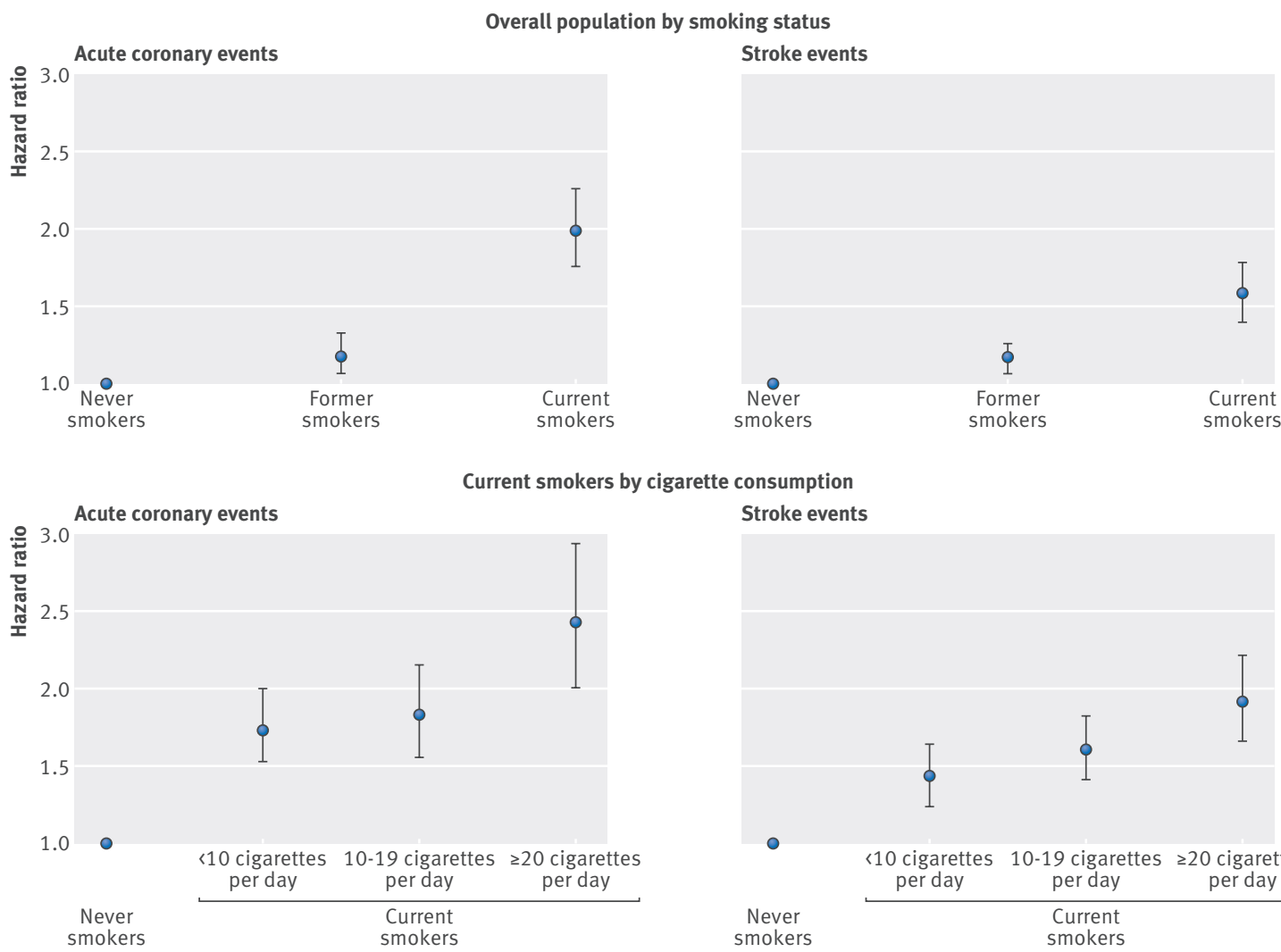

Former smokers by time since smoking cessation
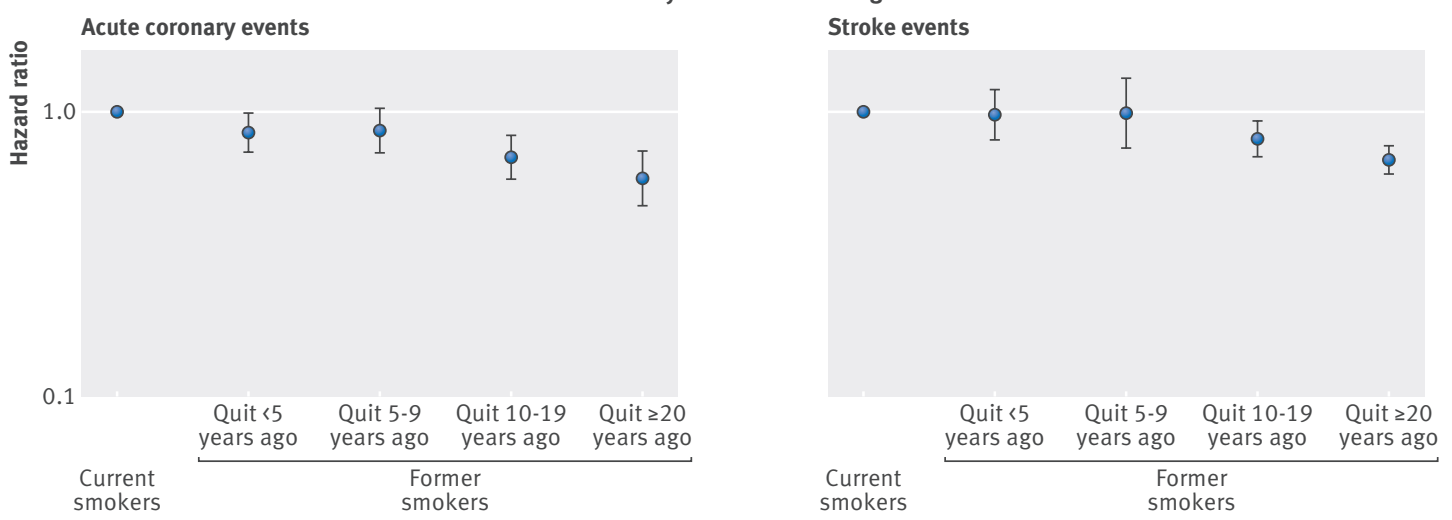

Fig 5 | Acute coronary events and stroke events summary estimates (random effects model) of hazard ratios for current smoking status, cigarette consumption and time since smoking cessation

the current smoking models $(40.0 \%$ for the former smoking models) for the age group 60 to 69 , and to $65.6 \%$ (52.9\%) for the age group 70 and older. NIH-AARP also contributed to heterogeneity between study results owing to its high statistical power that resulted in precise estimates with narrow confidence intervals. This reduced the extent of overlap in confidence intervals across studies, on which the $\mathrm{I}^{2}$ metric strongly depends, and thus inflated its value. ${ }^{30}$ Repeating the meta-analyses after exclusion of NIH-AARP accordingly led to substantially lower $\mathrm{I}^{2}$ values (56.2\% and 58.6\%) and shifted the summary estimates of the fixed effect model (for example, hazard ratio 2.02 (1.82 to 2.25) for current smoking and cardiovascular mortality, and 1.35 (1.23 to 1.49) for former smoking) towards the results of the random effects model. Acknowledging the statistical heterogeneity across the included cohort studies, we decided to follow a conservative approach and to report summary estimates of the random effects models as main results.

Some further limitations of our study need to be considered. Although all data were harmonised based on consented harmonisation rules, the data from the different cohort studies are still not perfectly comparable owing to differences in study design and data collection procedures, and to inconsistencies in definitions of variables. In addition, some cohorts did not have all variables available and models were thus fitted without these; however, sensitivity analyses by excluding these cohorts from the models did not change the summary 


\begin{tabular}{|c|c|c|c|c|c|c|c|}
\hline \multirow[b]{2}{*}{ Population } & \multirow[b]{2}{*}{ Smoking status } & \multicolumn{2}{|c|}{$\begin{array}{l}\text { Cardiovascular } \\
\text { deaths }\end{array}$} & \multicolumn{2}{|c|}{$\begin{array}{l}\text { Acute coronary } \\
\text { events }\end{array}$} & \multicolumn{2}{|c|}{ Stroke events } \\
\hline & & $\mathrm{HR}$ & $95 \% \mathrm{Cl}$ & $\mathrm{HR}$ & $95 \% \mathrm{Cl}$ & $\mathrm{HR}$ & $95 \% \mathrm{Cl}$ \\
\hline \multirow[t]{3}{*}{ Men } & Never smokers & 1.00 & & 1.00 & & 1.00 & \\
\hline & Former smokers & 1.33 & 1.20 to 1.48 & 1.18 & 1.00 to 1.38 & 1.08 & 0.97 to 1.21 \\
\hline & Current smokers & 1.95 & 1.69 to 2.25 & 1.80 & 1.51 to 2.15 & 1.44 & 1.23 to 1.68 \\
\hline \multirow[t]{3}{*}{ Women } & Never smokers & 1.00 & & 1.00 & & 1.00 & \\
\hline & Former smokers & 1.40 & 1.25 to 1.57 & 1.24 & 1.07 to 1.41 & 1.20 & 1.06 to 1.36 \\
\hline & Current smokers & 2.22 & 1.86 to 2.65 & 2.26 & 1.98 to 2.59 & 1.78 & 1.46 to 2.17 \\
\hline \multirow[t]{3}{*}{ Age $60-69$} & Never smokers & 1.00 & & 1.00 & & 1.00 & \\
\hline & Former smokers & 1.57 & 1.43 to 1.72 & 1.25 & 1.10 to 1.43 & 1.22 & 1.10 to 1.35 \\
\hline & Current smokers & 2.45 & 2.22 to 2.69 & 2.02 & 1.78 to 2.28 & 1.68 & 1.46 to 1.94 \\
\hline \multirow[t]{3}{*}{ Age 70+ } & Never smokers & 1.00 & & 1.00 & & 1.00 & \\
\hline & Former smokers & 1.21 & 1.08 to 1.36 & 1.12 & 0.95 to 1.32 & 1.10 & 0.95 to 1.28 \\
\hline & Current smokers & 1.70 & 1.42 to 2.04 & 1.88 & 1.41 to 2.52 & 1.49 & 1.22 to 1.82 \\
\hline
\end{tabular}

estimates to any meaningful extent. Irrespective of such drawbacks, our approach of conducting a meta-analysis with individual participant data from different cohort studies being harmonised and uniformly analysed can be presumed to be more valid compared to a traditional meta-analysis of published studies. ${ }^{31} \mathrm{~A}$ few other limitations might have contributed to an underestimation of the true associations of smoking with cardiovascular outcomes. Firstly, since we included only participants aged 60 and older in our study, heavier and long term smokers are probably under-represented in our sample owing to their increased mortality risks. Such a differential mortality bias would have led to an underestimation of risks for smokers. Secondly, we cannot rule out bias due to social desirability or imperfect recall, which could have led to under-reporting and thus to misclassification of current or former smokers to the never smoker category and thus to an underestimation of risk estimates. Thirdly, smoking was assessed at baseline only and repeated measurements of smoking behaviour over follow-up were not available. While smoking initiation is quite unlikely in older never smokers, smoking cessation in baseline smokers and relapses in former smokers are likely, leading to an underestimation of the effects of smoking and the benefits of quitting. ${ }^{32}$ The true associations of smoking and smoking cessation with cardiovascular outcomes are therefore probably stronger than observed in our study.

Particular strengths of our study are the focus on a previously understudied population, the inclusion of a large number of cohorts that cover a wide geographical area and the resulting broad generalisability of our results to older populations. Owing to the large sample size and accordingly high statistical power, we were further able to provide quite precise estimates for both hazard ratios and risk advancement periods.

\section{Implications and conclusions}

Even though comparably high quit success rates can be achieved, studies suggest that older smokers are less likely to receive smoking cessation support than younger smokers. ${ }^{33-35}$ Hence, our findings on the hazards of smoking and the benefits of quitting in older ages have important public health implications. Despite the attenuation of the relative risks with age, smoking cessation interventions in older adults could probably achieve even greater absolute reductions in adverse cardiovascular events than in younger or middle aged populations given the trends of population ageing in higher income countries and the higher incidence of cardiovascular events and mortality in older age. ${ }^{5}$ This tremendous potential for cardiovascular disease prevention will remain largely untapped unless tobacco prevention efforts in older people are intensified. Risk communication is especially crucial in promoting smoking cessation and risk advancement periods could be easier to grasp for the general public than other epidemiological risk measures such as relative risks or years of life lost. ${ }^{1314}$ In this study, we provided risk advancement period estimates for the association between smoking related variables and cardiovascular mortality. For example, we found a risk advancement period of 5.5 for cardiovascular mortality in current smokers in reference to never smokers, which denotes that death from cardiovascular disease is advanced by 5.5 years in the average current smoker above the age of 60 compared with the average never smoker of the same age. A risk advancement period of -1.34 for former smokers who stopped smoking 5 to 9 years ago (in reference to current smokers) denotes that these former smokers already "gained" 1.34 years through quitting and abstaining from smoking for at least five years. While we can only hypothesise that risk advancement periods might be a useful tool to convey the risks of smoking and the benefits of smoking cessation to smokers, we strongly encourage the realisation of experimental or intervention studies to evaluate the utility of risk advancement periods in the context of clinical or community programmes-for instance, by using the estimates that we provided in this study.

To conclude, our results corroborate and expand existing evidence that smoking is a strong independent risk factor of cardiovascular events and mortality even at older age, advancing cardiovascular mortality by more than five years. Importantly, our study reveals that even in older adults, smoking cessation is beneficial in reducing the excess risk caused by smoking. Given the current trends in demographic ageing, smoking cessation programmes should also focus on older people to curb the burden of smoking associated disease and mortality.

\section{AUTHOR AFFILIATIONS}

'Division of Clinical Epidemiology and Aging Research, German Cancer Research Center (DKFZ), Heidelberg, Germany ${ }^{2}$ Network Aging Research (NAR), University of Heidelberg, Heidelberg, Germany

${ }^{3}$ National Cancer Institute, Bethesda, MD, USA

${ }^{4}$ Department of Epidemiology and Public Health, University College London, London, UK

${ }^{5}$ Division of Human Nutrition, Wageningen University, Wageningen, Netherlands

${ }^{6}$ Centre for Health Protection, National Institute for Public Health and the Environment (RIVM), Bilthoven, Netherlands

7UKCRC Centre of Excellence for Public Health, Queen's University Belfast, Belfast, UK 
${ }^{8}$ National Institute for Health and Welfare (THL), Helsinki, Finland 9Institute of Public Health and Clinical Nutrition, University of Eastern Finland, Kuopio, Finland

${ }^{10}$ Hospital District of North Karelia, Joensuu, Finland

${ }^{11}$ Department for Determinants of Chronic Diseases (DCD), National Institute for Public Health and the Environment (RIVM), Bilthoven, Netherlands

${ }^{12}$ Department of Gastroenterology and Hepatology, University Medical Centre, Utrecht, Netherlands

${ }^{13}$ Department of Epidemiology and Biostatistics, The School of Public Health, Imperial College London, London, United Kingdom ${ }^{14}$ Department of Social and Preventive Medicine, Faculty of Medicine, University of Malaya, Kuala Lumpur, Malaysia

${ }^{15}$ Hellenic Health Foundation, Athens, Greece

${ }^{16}$ Department of Hygiene, Epidemiology and Medical Statistics, University of Athens Medical School, Athens, Greece

${ }^{17}$ Institute of Epidemiology II, Helmholtz Zentrum München, Neuherberg, Germany

${ }^{18} \mathrm{German}$ Center for Cardiovascular Disease Research (DZHK eV), partner-site Munich, Munich, Germany

${ }^{19}$ Department of Epidemiology, Julius Center for Health Sciences and Primary Care, University Medical Center Utrecht, Utrecht, Netherlands

${ }^{20}$ Department of Community Medicine, UiT The Arctic University of Norway, Tromsø, Norway

${ }^{21}$ Institute of Environmental Medicine, Karolinska Institutet, Stockholm, Sweden

${ }^{22}$ Institute for Translational Epidemiology and Tisch Cancer Institute, Icahn School of Medicine at Mount Sinai, New York, NY, USA

Contributors: CG, UM, and HB planned the study. UM carried out the statistical analyses, interpreted the data, and drafted the manuscript. $A M, B S$, and $H B$ contributed to the writing of the manuscript. All authors revised drafts critically for important intellectual content, and all authors reviewed and approved the final manuscript. UM is the guarantor.

Collaborators on behalf of the CHANCES consortium: Migle Baceviciene, Jolanda M A Boer, Wojciech Drygas, Sture Eriksson, Edith Feskens, Valeriy Gafarov, Julian Gardiner, Niclas Håkansson, Jan-Håkan Jansson, Pekka Jousilahti, Ellen Kampman, Jukka Kontto, Ruzena Kubinova, Max Leenders, Allan Linneberg, Maja-Lisa Løchen, Roberto Lorbeer, Sofia Malyutina, Ellisiv B Mathiesen, Håkan Melhus, Karl Michaëlsson, Inger Niølstad, Nicola Orsini, Andrzej Pająk, Hynek Pikhart, Charlotta Pisinger, Veikko Salomaa, María-José Sánchez, Susana Sans, Barbara Schaan, Andrea Schneider, Galatios Siganos, Stefan Söderberg, Martinette Streppel, Abdonas Tamošiūnas, Giovanni Veronesi, Eveline Waterham, Patrik Wennberg.

Funding: Data used throughout the present study are derived from the CHANCES project. The project is coordinated by the Hellenic Health Foundation, Greece. The project received funding by the FP7 framework programme of DG-RESEARCH in the European Commission (grant agreement no. HEALTH-F3-2010-242244). ELSA: The data of the ELSA cohort were made available through the UK Data Archive (UKDA). ELSA was developed by a team of researchers based at the NatCen Social Research, University College London and the Institute for Fiscal Studies. The data were collected by NatCen Social Research. The funding is provided by the National Institute of Aging in the United States, and a consortium of UK government departments coordinated by the Office for National Statistics. The developers and funders of ELSA and the archive do not bear any responsibility for the analyses or interpretations presented here. EPIC Greece: funded by the Hellenic Health Foundation. EPIC Netherlands: funded by European

Commission (DG SANCO); Dutch Ministry of Public Health, Welfare and Sports (VWS); The National Institute for Public Health and the Environment; the Dutch Cancer Society, the Netherlands Organisation for Health Research and Development (ZONMW); World Cancer Research Fund (WCRF). EPIC Spain: supported by Health Research Fund (FIS) of the Spanish Ministry of Health RTICC 'Red Temática de Investigación Cooperativa en Cáncer (grant numbers: Rd06/0020/0091 and Rd12/0036/0018), Regional Governments of Andalucía, Asturias, Basque Country, Murcia (project 6236), and Navarra, Instituto de Salud Carlos III, Redes de Investigacion Cooperativa (RD06/0020). EPIC Sweden: funded by the Swedish Cancer Society, the Swedish Scientific Council and the Regional Government of Skåne. ESTHER: funded by the Baden-Württemberg state Ministry of Science, Research and Arts (Stuttgart, Germany), the
Federal Ministry of Education and Research (Berlin, Germany), and the Federal Ministry of Family Affairs, Senior Citizens, Women and Youth (Berlin, Germany). HAPIEE: funded by the Wellcome Trust (064947 and 081081), the US National Institute on Aging (R01 AG23522-01) and a grant from Mac Arthur Foundation. MORGAM: MORGAM Project has received additional funding from European Union FP 7 projects ENGAGE (HEALTH-F4-2007-201413) and BiomarCaRE (278913). This has supported central coordination, workshops and part of the activities of the MORGAM Data Centre, at THL in Helsinki, Finland. MORGAM Participating Centres are funded by regional and national governments, research councils, charities, and other local sources. NIH-AARP: support for the National Institutes of Health (NIH)-AARP Diet and Health Study was provided by the Intramural Research Program of the National Cancer Institute (NCI), NIH. NHANES: The study is conducted by the National Center for Health Statistics (NCHS), Centers for Disease Control and Prevention. The findings and conclusions in this paper are those of the authors and not necessarily those of the agency. SENECA: SENECA is a Concerted Action within the EURONUT programme of the European Union. SHARE: SHARE data collection has been primarily funded by the European Commission through the 5th Framework Programme (project QLK6-CT-2001-00360 in the thematic programme Quality of Life), through the 6th Framework Programme (projects SHARE-I3, RII-CT-2006-062193, COMPARE, CIT5- CT-2005-028857, and SHARELIFE, CIT4-CT-2006-028812) and through the 7th Framework Programme (SHARE-PREP, No 211909, SHARE-LEAP, No 227822 and SHARE M4, No 261982). Additional funding from the US National Institute on Aging (U01 AG09740-13S2, P01 AG005842, P01 AG08291, P30 AG12815, R21 AG025169, Y1-AG-4553-01, IAG BSR06-11, and OGHA 04-064) and the German Ministry of Education and Research as well as from various national sources is gratefully acknowledged (see www. share-project.org for a full list of funding institutions). SMC: funded by the Swedish Research Council and Strategic Funds from Karolinska Institutet. Tromsø: funded by: UiT The Arctic University of Norway, the National Screening Service, and the Research Council of Norway. Zutphen Elderly Study: funded by the Netherlands Prevention Foundation. The work of Aysel Müezzinler was supported by a scholarship from the Klaus Tschira Foundation.

Competing interests: All authors have completed the ICMJE uniform disclosure form at http://www.icmje.org/coi_disclosure.pdf (available on request from the corresponding author) and declare: no support from any organisation for the submitted work; no financial relationships with any organisations that might have an interest in the submitted work in the previous three years; no other relationships or activities that could appear to have influenced the submitted work.

Ethics approval: The included studies have been approved by local ethic committees as follows: ELSA: UK National Research Ethics Committee. EPIC: Ethics Committee of the International Agency for Research on Cancer and at each participating centre (EPIC Greece: ethics committees of the University of Athens Medical School and the Hellenic Health Foundation; EPIC the Netherlands: Institutional Review Board of the University Medical Center Utrecht (EPIC Utrecht) and the Medical Ethical Committee of TNO Nutrition and Food Research (EPIC Bilthoven); EPIC Spain: Clinical Research Ethics Committee (CEIC); EPIC Sweden: Research Ethics Committee of Umeå University). ESTHER: Medical Faculty of the University of Heidelberg and the Medical Board of the state of Saarland. HAPIEE: Ethics committees at University College London and at each participating centre (HAPIEE Czech Republic: National Institute of Public Health, Prague; HAPIEE Poland: Collegium Medicum, Jagiellonian University, Krakow; HAPIEE Russia: Institute of Internal Medicine, Siberian Branch of the Russian Academy of Medical Sciences, Novosibirsk; HAPIEE Lithuania: Institute of Cardiology, Lithuanian University of Health Sciences, Kaunas). MORGAM Brianza: Ethics Committee, Monza Hospital. MORGAM Catalonia: Ethics board of the Institute of Health Studies, Barcelona. MORGAM FINRISK: 1980s: no ethics approval required for observational studies (but current laws allow the use of these data for public health research), 1990s: Ethics committee of the National Public Health Institute (KTL), 2002: Ethics Committee of Epidemiology and Public Health in Hospital District of Helsinki and Uusimaa. MORGAM Glostrup: Ethics Committee of the Capital Region (formerly Copenhagen County), Denmark. MORGAM KORA Augsburg: Local authorities and the Bavarian State Chamber of Physicians. MORGAM Northern Sweden: Research Ethics Committee of Umeå University. MORGAM SHIP Greifswald: Human research ethics committee of the Medical School at the University of Greifswald. MORGAM Warsaw: Warsaw Medical University Ethical Board. NHANES: CDC/NCHS Ethics Review Board. NIH-AARP: Special Studies Institutional Review Board of the NCI; SENECA: Local ethics approval was obtained by the SENECA participating centres. SHARE: Ethics Committee of the University of 
Mannheim. SMC: Regional Ethical Board at Karolinska Institutet, Stockholm. Tromsø: Regional Committee for Medical and Health Research Ethics and the Data Inspectorate of Norway. Zutphen Elderly Study: Medical Faculty, University Leiden and Ethics Committee of the Netherlands Organisation for Applied Scientific Research (TNO).

The lead author and guarantor of this study affirms that this manuscript is an honest, accurate, and transparent account of the study being reported; that no important aspects of the study have been omitted; and that any discrepancies from the study as planned have been explained.

Independence of researchers from funders: The study's funders played no role in study design; in the collection, analysis, and interpretation of data; in the writing of the report; and in the decision to submit the article for publication. The researchers were independent of the funders.

This is an Open Access article distributed in accordance with the terms of the Creative Commons Attribution (CC BY 4.0) license, which permits others to distribute, remix, adapt and build upon this work, for commercial use, provided the original work is properly cited. See: http:// creativecommons.org/licenses/by/4.0/

1 US Department of Health, Education, and Welfare. Smoking and Health: Report of the Advisory Committee to the Surgeon General of the Public Health Service. Washington, DC: US Public Health Service. Office of the Surgeon General, 1964.

2 US Department of Health and Human Services. The health consequences of smoking -50 years of progress. A Report of the Surgeon General. Atlanta, GA: US Department of Health and Human Services, Centers for Disease Control and Prevention, National Center for Chronic Disease Prevention and Health Promotion, Office on Smoking and Health, 2014

3 Doll R, Peto R, Boreham I. Sutherland I. Mortality in relation to smoking: 50 years' observations on male British doctors. BMJ 2004:328:1519

4 Ford ES, Capewell S. Proportion of the decline in cardiovascular mortality disease due to prevention versus treatment: public health versus clinical care. Annu Rev Public Health 2011;32:5-22.

5 Kriekard P, Gharacholou SM, Peterson ED. Primary and secondary prevention of cardiovascular disease in older adults: a status report. Clin Geriatr Med 2009;25:745-55.

6 Beer C, Alfonso H, Flicker L, Norman PE, Hankey GJ, Almeida OF. Traditional risk factors for incident cardiovascular events have limited importance in later life compared with the health in men study cardiovascular risk score. Stroke 2011;42:952-9.

7 Gellert C, Schöttker B, Müller H, Holleczek B, Brenner H. Impact of smoking and quitting on cardiovascular outcomes and risk advancement periods among older adults. EurJ Epidemiol 2013;28:649-58.

8 Iso H, Date C, Yamamoto A, Toyoshima H, Watanabe Y, Kikuchi S, et al. Smoking cessation and mortality from cardiovascular disease among Japanese men and women: the JACC Study. Am J Epidemiol 2005;161:170-9.

9 Kelly TN, Gu D, Chen J, Huang JF, Chen JC, Duan X, et al. Cigarette smoking and risk of stroke in the Chinese adult population. Stroke 2008;39:1688-93.

10 Myint PK, Sinha S, Luben RN, Bingham SA, Wareham NJ, Khaw KT. Risk factors for first-ever stroke in the EPIC-Norfolk prospective population-based study. Eur / Cardiovasc Prev Rehabil 2008;15:663-9.

11 Parish S, Collins R, Peto R, Youngman L, Barton J, Jayne K, et al. Cigarette smoking, tar yields, and non-fatal myocardial infarction: 14000 cases and 32000 controls in the United Kingdom. The International Studies of Infarct Survival (ISIS) Collaborators. BM/1995;311:471-7.

12 Thun MJ, Carter BD, Feskanich D, Freedman ND, Prentice R, Lopez AD, et al. 50-year trends in smoking-related mortality in the United States. N Engl J Med 2013;368:351-64

13 Gellert C, Schöttker B, Holleczek B, Stegmaier C, Müller H, Brenner $\mathrm{H}$. Using rate advancement periods for communicating the benefits of quitting smoking to older smokers. Tob Control 2013;22:227-30.
14 Liese AD, Hense HW, Brenner H, Löwel H, Keil U. Assessing the impact of classical risk factors on myocardial infarction by rate advancement periods. Am / Epidemiol 2000;152:884-8.

15 Brenner H, Gefeller O, Greenland S. Risk and rate advancement periods as measures of exposure impact on the occurrence of chronic diseases. Epidemiol 1993:4:229-36.

16 Boffetta P, Bobak M, Borsch-Supan A, Brenner H, Eriksson S, Grodstein F, et al. The Consortium on Health and Ageing: Network of Cohorts in Europe and the United States (CHANCES) project-design, population and data harmonization of a large-scale, international study. Eur ! Epidemiol 2014;29:929-36.

17 Mendis S, Thygesen K, Kuulasmaa K, Giampaoli S, Mähönen M, Ngu Blackett K, et al. World Health Organization definition of myocardia infarction: 2008-09 revision. Int J Epidemiol 2011;40:139-46.

18 Tunstall-Pedoe H, Kuulasmaa K, Amouyel P, Arveiler D, Rajakangas AM, Pajak A. Myocardial infarction and coronary deaths in the World Health Organization MONICA Project. Registration procedures, event rates, and case-fatality rates in 38 populations from 21 countries in four continents. Circulation 1994;90:583-612.

19 World Cancer Research Fund. Food, Nutrition, Physical Activity, and the Prevention of Cancer: a Global Perspective. Washington, DC: American Institute for Cancer Research, 2007.

20 DerSimonian R, Laird N. Meta-analysis in clinical trials. Control Clin Trials 1986;7:177-88.

21 Higgins JP, Thompson SG. Quantifying heterogeneity in a metaanalysis. Stat Med 2002;21:1539-58.

22 Greenland S, Longnecker MP. Methods for trend estimation from summarized dose-response data, with applications to meta-analysis. Am J Epidemiol 1992;135:1301-9

23 Orsini N, Li R, Wolk A, Khudyakov P, Spiegelman D. Meta-analysis for linear and nonlinear dose-response relations: examples, an evaluation of approximations, and software. Am J Epidemiol 2012;175:66-73.

24 Schwarzer G. meta: Meta-Analysis with R. http://CRAN.R-project.org/ package=meta, 2013

25 Tournier M, Moride Y, Lesk M, Ducruet T, Rochon S. The depletion of susceptibles effect in the assessment of burden-of-illness: the example of age-related macular degeneration in the community-dwelling elderly population of Quebec. Can J Clin Pharmacol 2008;15:e22-35.

26 Kenfield SA, Wei EK, Rosner BA, Glynn RJ, Stampfer MJ, Colditz GA. Burden of smoking on cause-specific mortality: application to the Nurses' Health Study. Tob Control 2010;19:248-54.

27 Pirie K, Peto R, Reeves GK, Green J, Beral V, Million Women Study Collaborators. The 21st century hazards of smoking and benefits of stopping: a prospective study of one million women in the UK. Lancet 2013;381:133-41.

28 Huxley RR, Woodward M. Cigarette smoking as a risk factor for coronary heart disease in women compared with men: a systematic review and meta-analysis of prospective cohort studies. Lancet 2011;378:1297-305

29 Peters SA, Huxley RR, Woodward M. Smoking as a risk factor for stroke in women compared with men: a systematic review and meta-analysis of 81 cohorts, including 3,980,359 individuals and 42,401 strokes. Stroke 2013;44:2821-8.

30 Higgins JP. Commentary: Heterogeneity in meta-analysis should be expected and appropriately quantified. Int J Epidemio/ 2008:37:1158-60.

31 Riley RD, Lambert PC, Abo-Zaid G. Meta-analysis of individual participant data: rationale, conduct, and reporting. BMJ 2010;340:c221.

32 He Y, Jiang B, Li LS, Li LS, Sun DL, Wu L, et al. Changes in smoking behavior and subsequent mortality risk during a 35-year follow-up of a cohort in Xi'an, China. Am J Epidemiol 2014;179:1060-70.

33 Maguire CP, Ryan J, Kelly A, O'Neill D, Coakley D, Walsh JB. Do patient age and medical condition influence medical advice to stop smoking? Age Ageing 2000;29:264-6.

34 Buckland A, Connolly MJ. Age-related differences in smoking cessation advice and support given to patients hospitalised with smoking-related illness. Age Ageing 2005;34:639-42.

35 Doolan DM, Froelicher ES. Smoking cessation interventions and older adults. Prog Cardiovasc Nurs 2008;23:119-27.

(C) BMJ Publishing Group Ltd 2015

Appendix 